\title{
The impact of weather conditions on patterns of facial fractures
}

\begin{abstract}
Purpose: Facial fractures account for over 400,000 emergency room visits and nearly 1 billion dollars in yearly health care costs. ${ }^{1}$ Understanding patterns of facial fracture volume can inform resource allocation and improve patient outcomes.
\end{abstract}

Patients and methods: Retrospective chart review was performed at a busy level 1 trauma center looking at facial fracture presentation data from 2010-2014 were reviewed in relation to weather, temperature, and other variables. Regional weather data was obtained from online sources. Temperature, humidity, precipitation, and other patterns were correlated to trauma incidence using a multivariate logistic regression model. Records from 3,666 facial fracture patients spanning 5 years were evaluated.

Results: $53 \%$ of all fractures occurred in patients aged $20-40$ years old. $77 \%$ of facial fractures were in males. Weekend days, the summer season, and the month of fracture presentation were all significantly associated with facial fracture incidence. Patients under 60 years of age were significantly more likely to be male. There was no correlation between phases of the moon and trauma incidence.

Conclusion: Facial fractures occurred most frequently in males aged 20-40 years old at our center. Several significant statistical associations between temporal, seasonal, and weather parameters were correlated with trauma incidence.

Keywords: facial fractures, weather, mandible fracture
Volume 10 Issue $3-2018$

\author{
Jordan Malenke,' Rachel Georgopoulos,' \\ Dahaoi Yu, ${ }^{2}$ Jeffrey C Liu' \\ 'Department of Otolaryngology-Head \& Neck Surgery, Temple \\ University School of Medicine, USA \\ ${ }^{2}$ Department of Clinical Science, Temple University School of \\ Medicine, Philadelphia, USA
}

Correspondence: Jeffrey C Liu, Department of

Otolaryngology, Temple University School of Medicine, 3440 N. Broad Street, Kresge West \#300, Philadelphia, Pennsylvania 19140, USA, Email jeffrey.liu@temple.edu

Received: December 29, 2017 | Published: June 22, 2018

\section{Introduction}

Trauma related injuries remain a major public health problem in the United States. One in five trauma patients will have an associated facial trauma. ${ }^{2}$ In 2007 a total of 407,167 emergency room visits in the U.S. were attributable to facial fractures. ${ }^{1}$ The financial burden associated with the care of facial trauma is quite significant. In 2007 close to $\$ 1$ billion dollars was spent on facial fracture related emergency department visits alone. ${ }^{1}$ The significant discordance between the cost of care among facial fracture patients and the low reimbursement for services rendered lends to difficulties with providing incentives for subspecialty coverage of facial trauma and to a significant financial burden to level one trauma centers nationwide. ${ }^{2,3}$ Characterization of variables that affect trauma volume can allow for appropriate resource allocation

While the effects of season on trauma incidence has been reported in the literature, information regarding temporal variance patterns and facial fracture volume is not as well characterized. ${ }^{1}$ It has been found that hip fractures occur more frequently in the winter months while myocardial infarctions, cardiac arrests and adult forearm fractures occur more frequently on days with snowfall..$^{2-5}$ Days with increased sunshine have been associated with increases in pediatric radius fractures. ${ }^{6}$ It has been anecdotally noted that facial trauma appears to be associated with warm temperatures or phases of the moon, but few studies have examined this specifically. We present a single institution experience of facial fracture presentations over five years at a busy level 1 trauma center in the Northeast, a region that experiences a variety of weather patterns.

\section{Materials and methods}

Medical records from Temple University Hospital were collected using the ICD-9 codes pertaining to facial fractures. Facial lacerations or blunt injuries were not included. Using these codes, a database of facial fracture presentations spanning all hospital presentations from 2010 to 2014 was populated.

Publically available weather data using National Weather Service (NWS) data recorded at the local Northeast Philadelphia Airport (KPNE) were downloaded via Weather Underground. ${ }^{7}$ Northeast Philadelphia Airport is 8.7 miles from our hospital. This provided weather data for all days contained within the dataset. The daily weather variables recorded included minimum temperature, maximum temperature, mean temperature, dew points, maximum humidity, minimum humidity, mean humidity, quantity of precipitation, and wind speed among other variables. Publically available lunar phase data, recorded by the U.S. Naval observatory, was accessed using Somacon. ${ }^{8}$

Facial fracture presentation data was then paired to the weather and lunar variables corresponding to the day of presentation. Statistical analyses were performed using Microsoft Excel and SAS. Pertinent statistical tests included Wilcoxon's ranked sums, Fischer or chisquare test, and Mann-Whitney's U-test.

\section{Results}

3,666 unique patients with facial fractures from 2010 to 2014 at a single level 1 trauma were included in this study. Males accounted for $77 \%$ of the patients (Table 1). The majority of our patients were adults less than forty years of age. (Figure 1) The mean age for all patients was 36.8 years of age. The mean age for male patients was 35.9 years, and the mean age for females was 39.9 years. 54\% of all patients admitted with facial fractures were between ages 20 and 39 . By age 70 the gender ratio normalized. 
Table I Demographics of Facial fracture Patients

\begin{tabular}{lll}
\hline Domain & Study $\mathbf{n}$ & (\%) \\
\hline Age, mean & 37.6 & \\
Population Sex: & & \\
Male & 2821 & $-77 \%$ \\
Female & 845 & $-23 \%$ \\
Race: & & \\
African American/Black & 1484 & $-40.50 \%$ \\
Caucasian White & 1053 & $-29 \%$ \\
Hispanic & 928 & $-25 \%$ \\
Asian & 22 & $-0.60 \%$ \\
Other & 128 & $-3.50 \%$ \\
Unknown & 51 & $-1.40 \%$ \\
\hline
\end{tabular}

The daily average facial fracture presentation rate was about 2.01 per day. There was a seasonal trend in presentations, with an increase in average daily presentation frequency during the summer months, and a decrease in presentations during the winter months. (Figure 2) (Figure 3) The highest average daily volume was in September, with 2.41 presentations. January and December had the lowest average daily volume with 1.54 and 1.72 , respectively. The difference between facial fracture presentations in the summer and winter season, as well as the months of August, September, and October was statistically significant from the other seasons/months $(\mathrm{p}<0.0001)$.

The day of the week was also found to have a significant association to facial fracture volume, with weekends having an increase in average daily trauma volume. Weekend days had an average of 2.23 presentations per day while weekdays had an average of 1.92 presentations per day $(p<0.0001)$ (Figure 4). Phases of the moon were not found to have any significant effect on the average daily trauma volume $(\mathrm{p}=0.7136)$. The average daily incidence was 1.86 during the new moon phase, 2.03 during the first quarter, 2.06 during the full moon phase, and 2.08 during the last quarter of the cycle. This was not significantly different.

There was no association between precipitation and facial fracture incidence. $(p=0.3298)$ As the daily maximum temperature rose, there was an increase in the rate of facial fracture presentations until temperatures reached 90 degrees F. $(p<0.0001)$ (Figure5) (Figure 6).

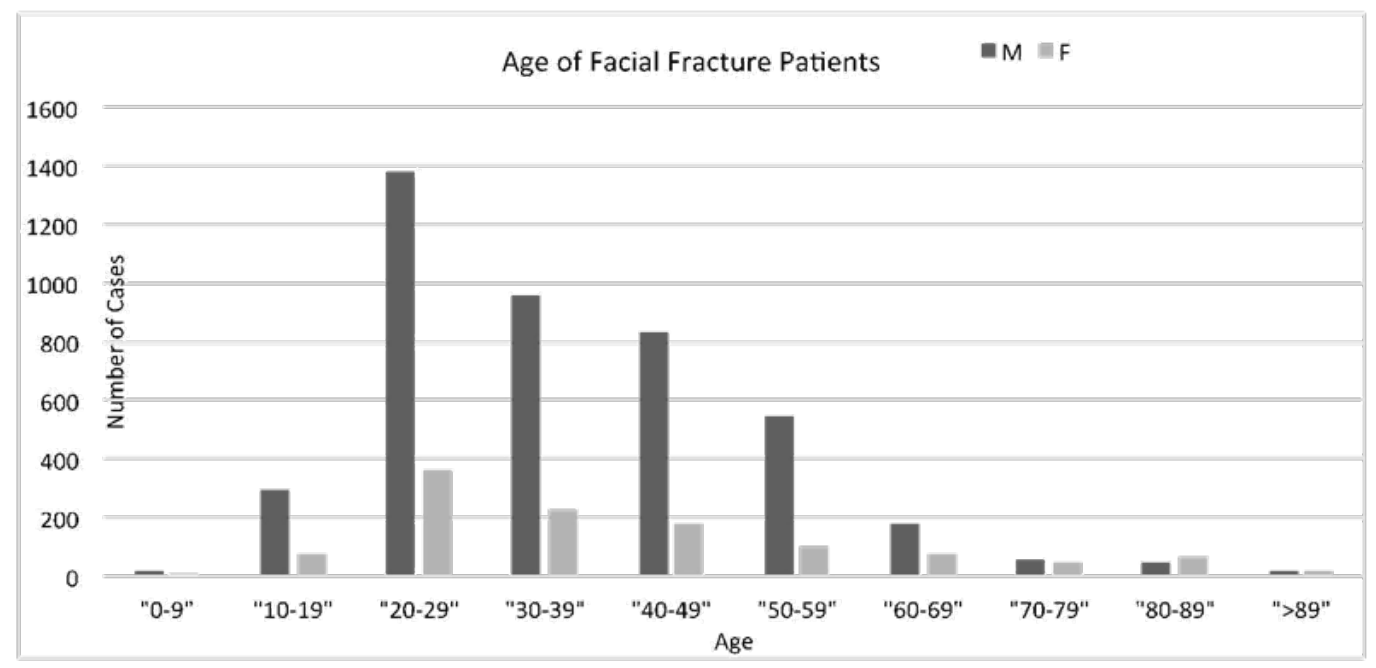

Figure I Gender and age distribution of facial fracture patients.

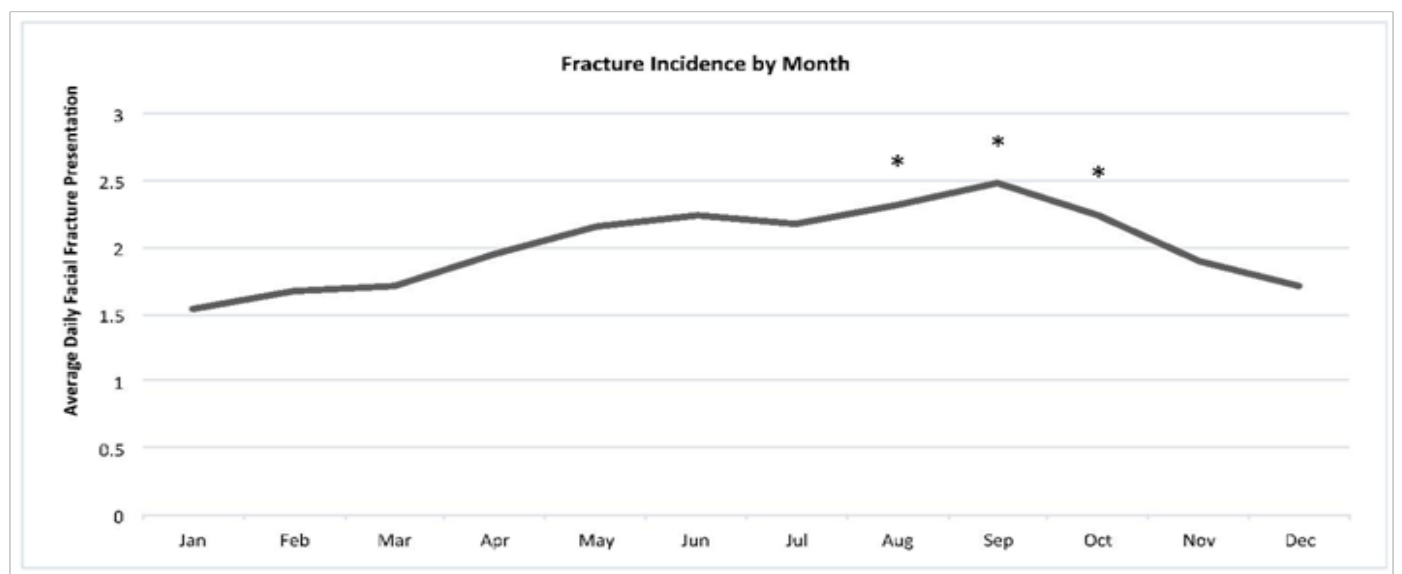

Figure 2 Average daily facial fracture presentations by month of the year.August, September, and October were found to be statistically significantly different from all other months of the year by chi square testing. 


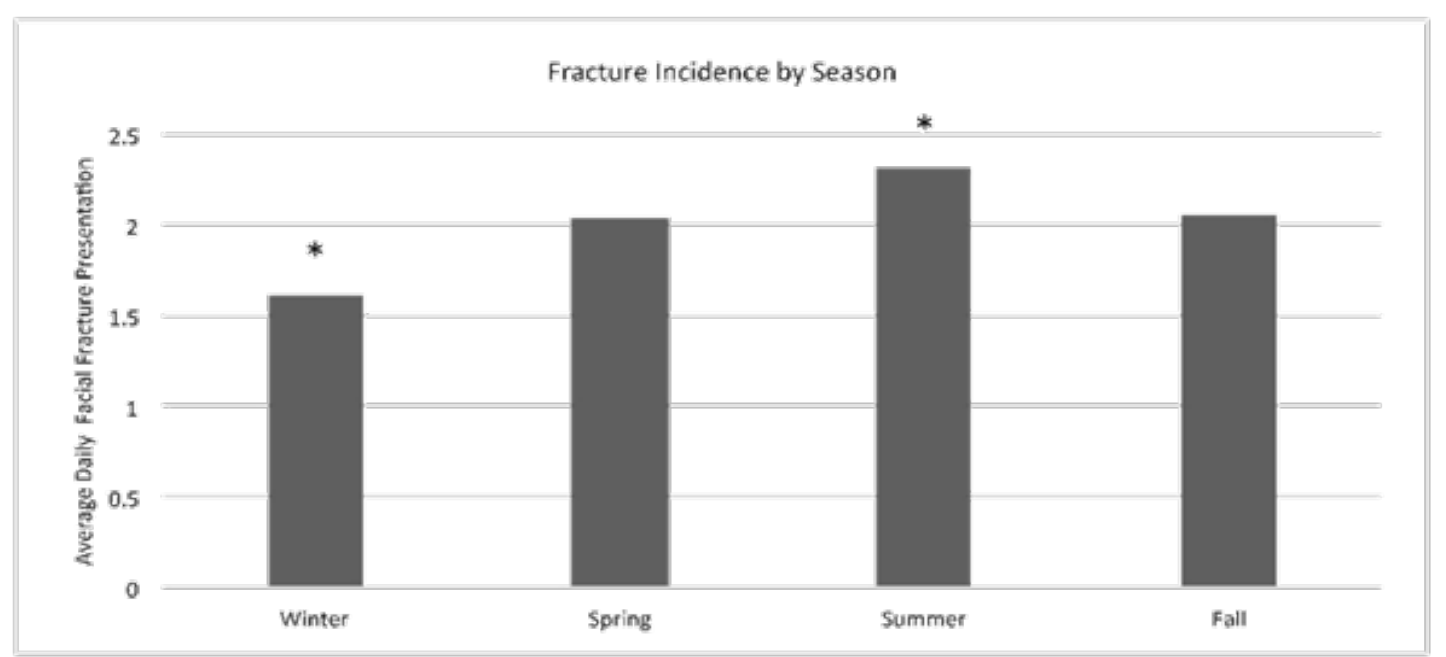

Figure 3 Average daily facial fracture presentations by season of the year.* Winter and summer had significant difference in average daily presentations as compared to all other months by chi square testing.

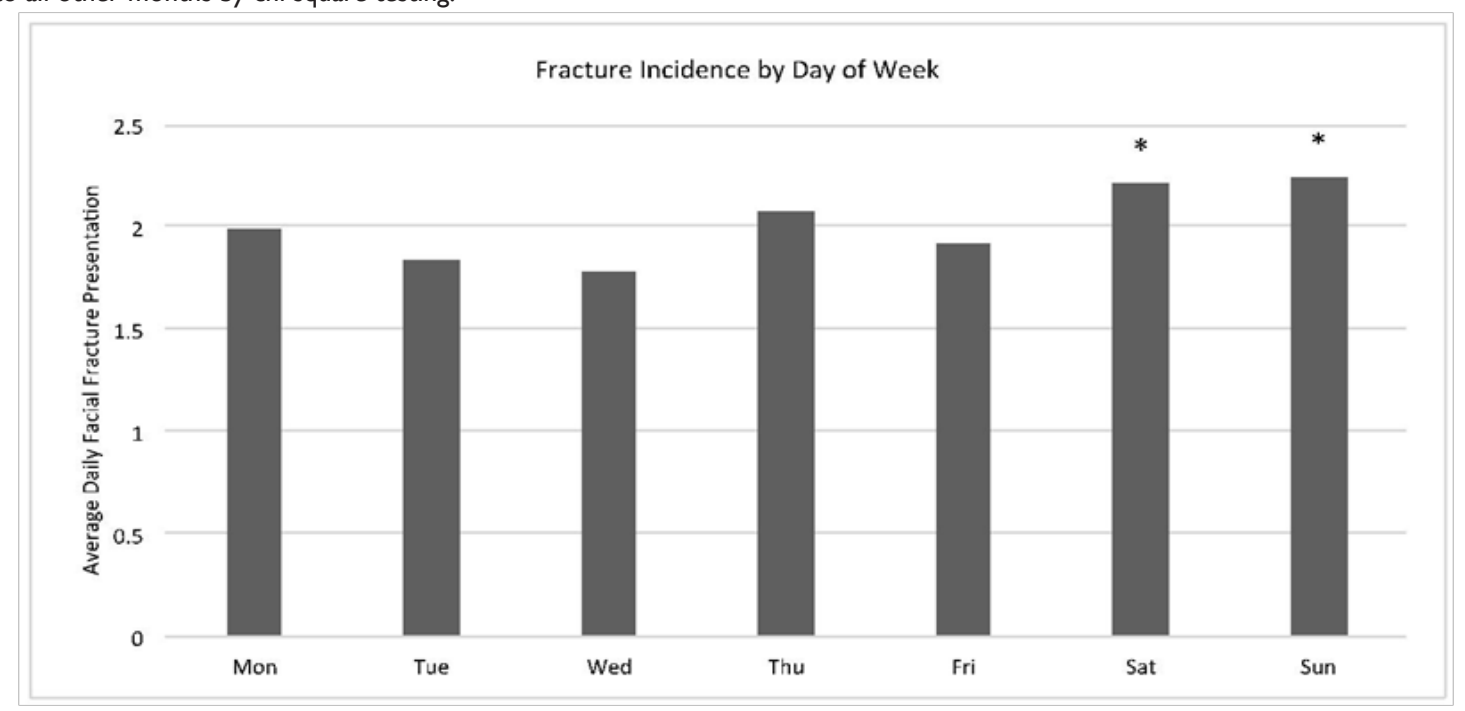

Figure 4 Average daily facial fracture presentations shown by day of the week. *Saturday and Sunday had a statistically significant increase in average daily presentations as compared to all other days by chi square testing.

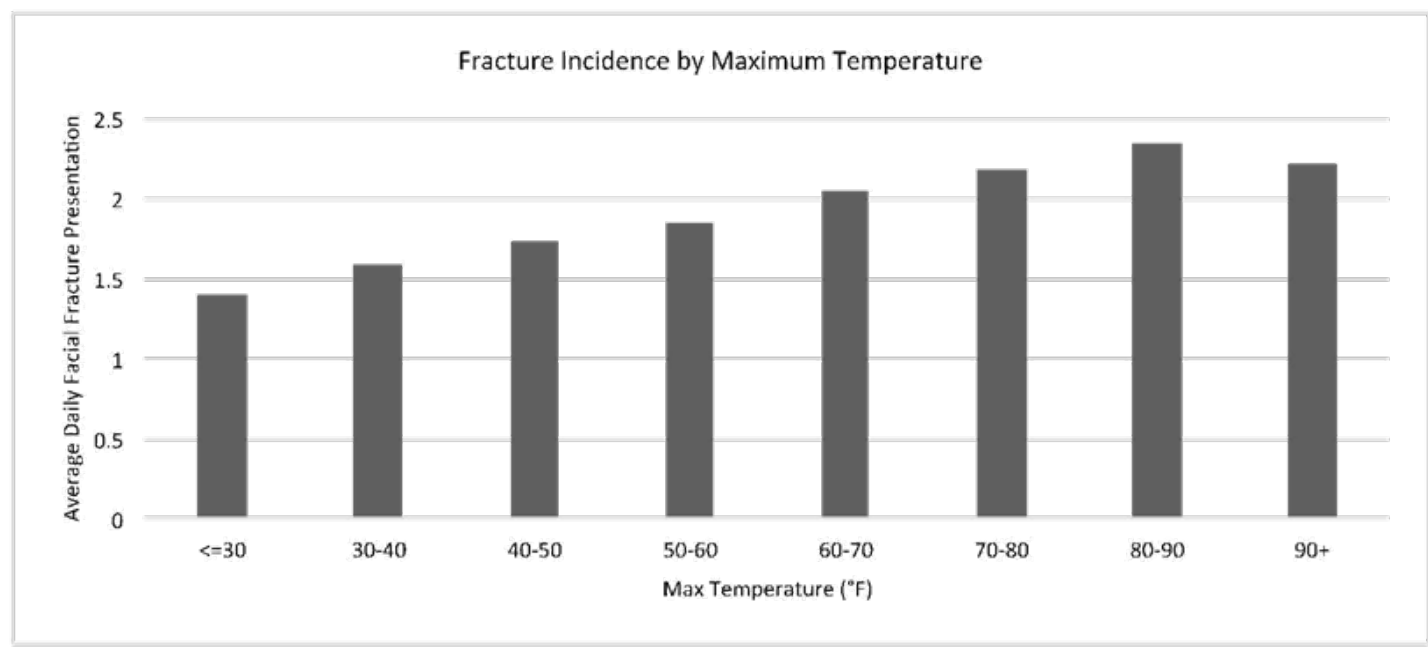

Figure 5 Average daily facial fracture presentations by daily maximum temperature. 


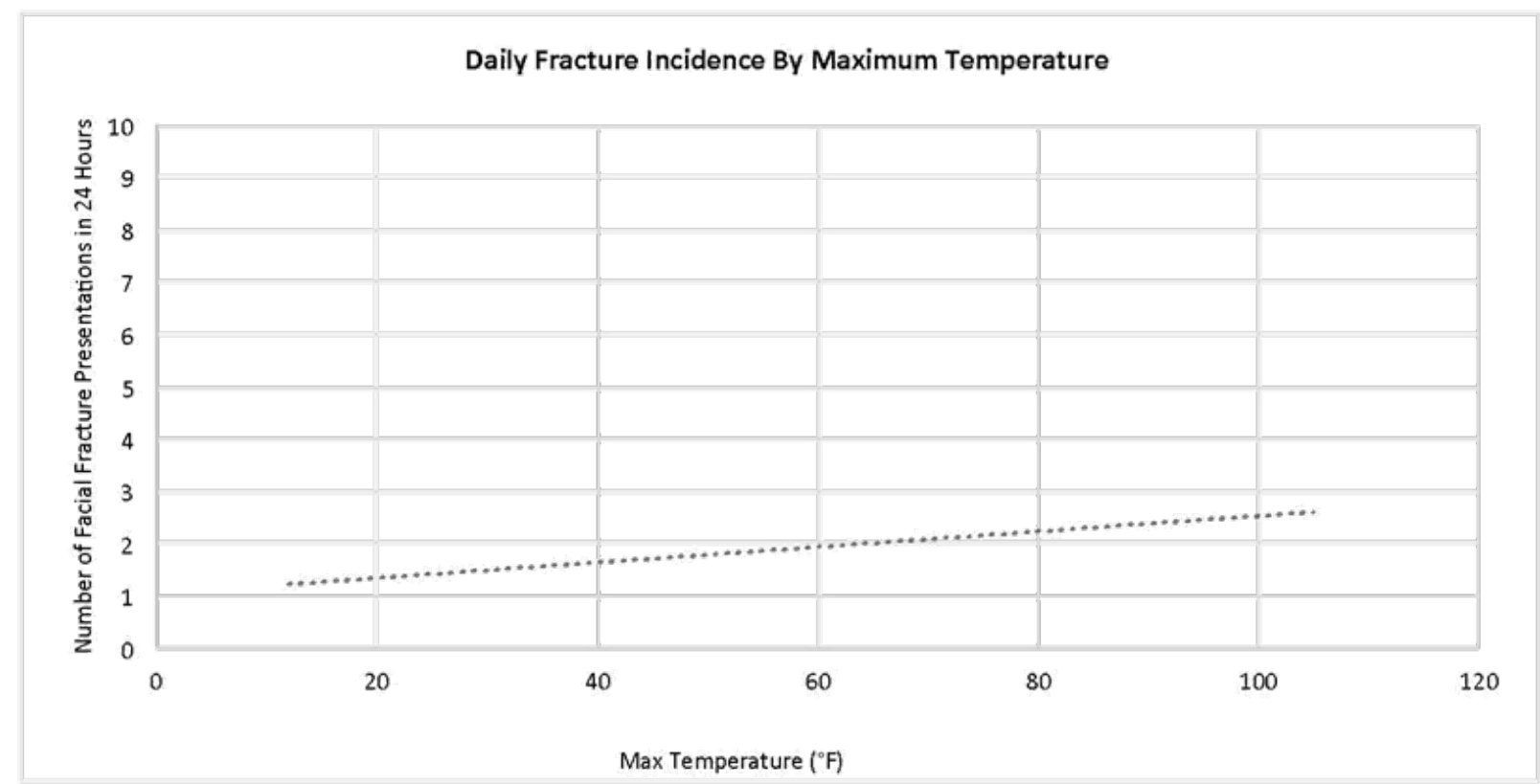

Figure 6 Scatterplot showing the daily total facial fracture admissions by daily maximum temperature.

\section{Discussion}

While the association between weather patterns and general trauma occurrence has been studied, until now the association with facial fracture specific presentations and weather patterns was less well defined. In accordance with the general trauma admission data, this study demonstrated that there is an increase in average daily presentations during the summer months and a decrease in facial fracture presentations during the winter season. ${ }^{9-11}$ Furthermore, increasing ambient temperatures were associated with an increase in the average daily fracture volume until a high temperature of $90^{\circ} \mathrm{F}$. The highest average daily volume occurred when the temperature was between 80 and $90^{\circ} \mathrm{F}$, with an average of 2.35 presentations. In contrast, average daily presentations were lowest when the temperature was below $30^{\circ} \mathrm{F}$ with an average of 1.40 . Facial fracture volume was statistically more significant on weekends when compared to weekdays, 2.23 and 1.92 respectively $(\mathrm{p}<0.0001)$. These results likely reflect that facial fracture volume increases when weather conditions are optimal for people to partake in outdoor activities.

While we hypothesized that precipitation and phase of the moon would affect facial fracture occurrence, neither of these variables had a statistically significant impact on facial fracture volume. In the United States, the most common causes of facial fracture admissions to emergency departments are assaults $(37 \%)$, falls $(25 \%)$, followed by motor vehicle collisions (MVCs) $(12 \%) .{ }^{1}$ While the number of facial fractures attributable to MVCs may increase with inclement weather conditions, this may not be reflected in our analysis of our center. This study did not capture mechanism of trauma, which will be addressed in a future study. Further analysis needs to be done in order to better characterize how weather patterns affect facial fracture volume by mechanism of injury.
Data obtained from this study was limited to a single Level 1 trauma center in northeast United States. It is also representative of an inner city urban tertiary medical center which serves a high number of Medicaid patients. Extrapolating this data to other major centers is limited by different patient demographics and typical weather patterns for different regions. Further studies need to be performed in order to study the associations between weather patterns and the mechanism of injury for facial fractures.

\section{Conclusion}

Weather patterns play a significant role in our activities and consequently it has a profound impact on the incidence of facial fracture. At our academic medical center in the Northeast, there is an increase in facial fracture presentations during the weekends, during the summer months, and during days with warmer temperatures up to $90^{\circ} \mathrm{F}$. Weather patterns influence our behaviors and have a role in the amount of facial fractures that present to our institution. Awareness of these trends can allow care providers to be better equipped to provide for such patients.

\section{Authorship}

Jordan Malenke, conception and study design, analysis and interpretation of data, drafting and revision of the manuscript; Rachel Georgopoulos, acquisition, analysis and interpretation of the data, drafting and revision of the manuscript; Daohai Yu, statistical analysis and interpretation of the data; Jeffrey C. Liu, conception and study design, acquisition, analysis and interpretation of the data, drafting and revision of the manuscript.

\section{Acknowledgments}

None. 


\section{Conflict of interest}

The author declares there is no conflict of interest

\section{References}

1. Allareddy V, Allareddy V, Nalliah RP. Epidemiology of facial fracture injuries. J Oral Maxillofac Surg. 2011;69(10):2613-2618.

2. Osman MF, Baugh RF, Baugh AD, et al. Relative value units and payer mix analysis of facial trauma coverage at a level 1 trauma center: is the current model sustainable? Surgery. 2014;156(4):995-1000.

3. Abubaker AO, Lynam GT. Changes in charges and costs associated with hospitalisation of patients with mandibular fractures between 1991 and 1993. J Oral Maxillofac Surg. 1998;56(2):161-167.

4. Shiue I, Muthers S, Bearman N. The role of cold stress in predicting extra cardiovascular and respiratory admissions. Int $J$ Cardiol. 2014;172(1):e109-e110.
5. Southern D, Knudtson ML, Ghali W. Myocardial infarction on snow days: incidence, procedure, use and outcomes. Can J Cardiol. 2006;22(1):5961.

6. Masterson E, Borton D, O'Brien T. Victims of our climate. Injury. $1993 ; 24(4): 247-248$.

7. https://www.wunderground.com/history/airport/KPNE

8. https://www.somacon.com/p570.php

9. Bhattacharyya T, Millham FH. Relationship between weather and seasonal factors and trauma admission volume at a Level I trauma center. J Trauma. 2001;51(1):118-122.

10. Atherton WG, Harper WM, Abrams KR. A year's trauma admissions and the effect of the weather. Injury. 2005;36(1):40-46.

11. Parsons N, Odumenya M, Edwards A, et al. Modelling the effects of the weather on admissions to UK trauma units: a cross-sectional study. Emerg Med J. 2011;28(10):851-855. 